\title{
ESTADO DEL ARTE DEL AGUA Y SANEAMIENTO RURAL EN COLOMBIA
}

\section{State of the art of water and rural sanitation in Colombia}

\author{
William Carrasco Mantilla \\ Consultor Internacional en Agua y Saneamiento, Universidad de los Andes. Contacto: wcarrasco2002@yahoo.com
}

\section{Resumen}

En la actualidad, más 11 millones de colombianos habitan en el campo. Sin embargo, una tercera parte de esta población no tiene acceso a agua potable ni a soluciones adecuadas de saneamiento básico. El balance de los Objetivos de Desarrollo del Milenio (ODM) deja mal parado el sector de agua y saneamiento rural en Colombia: según las cifras del Joint Monitoring Program, el país no cumplió con las metas pactadas para el 2015, y de continuar con esta tendencia en materia de inversiones y apoyo institucional para el área rural, tampoco se espera que se cumplan las nuevas metas pactadas para el año 2030 en los Objetivos de Desarrollo Sostenible (ODS).

Palabras clave: Agua potable, saneamiento rural básico, Objetivos del Milenio, posconflicto.

\section{Antecedentes}

El sector de agua potable y saneamiento básico rural en Colombia ha pasado por diferentes etapas en las últimas cinco décadas: Entre 1968 y 1987, la División de Saneamiento Básico Rural del Instituto Nacional de Salud (INS) desarrolló el Programa de Agua Potable y Saneamiento Básico Rural (PASBR), dirigido a comunidades de menos de 2.500 habitantes, para asistirlas en la construcción, operación y mantenimiento de sistemas de abastecimiento de agua y saneamiento de bajo costo.

\section{Abstract}

Currently, over 11 million Colombians live in the countryside. However, one third of the population has no access to safe water or adequate sanitation solutions básico.El balance of the Millennium Development Goals (MDGS) leaves the sector badly rural water and sanitation in Colombia: as figures from the Joint Monitoring Program, the country failed to meet the targets agreed for 2015, and to continue this trend in investment and institutional support for rural areas, it is not expected that the new targets agreed for 2030 are met in Sustainable Development Goals (ODS).

Keywords: Clean water, Basic rural sanitation, Millennium Development Goals, Postconflict.
Durante este periodo, la financiación de las obras se cubría en un 15\% por parte de las juntas comunitarias, un $45 \%$ a cargo de los municipios o departamentos y el $40 \%$ restante mediante crédito del Gobierno Nacional a 15 años y 6\% de interés anual. Para la operación del sistema, cada comunidad encargaba a uno de sus miembros — remunerado a medio tiempo - para llevar la contabilidad, controlar la calidad del agua y realizar reparaciones menores, previo adiestramiento del INS (entidad que continuaba brindando apoyo durante 


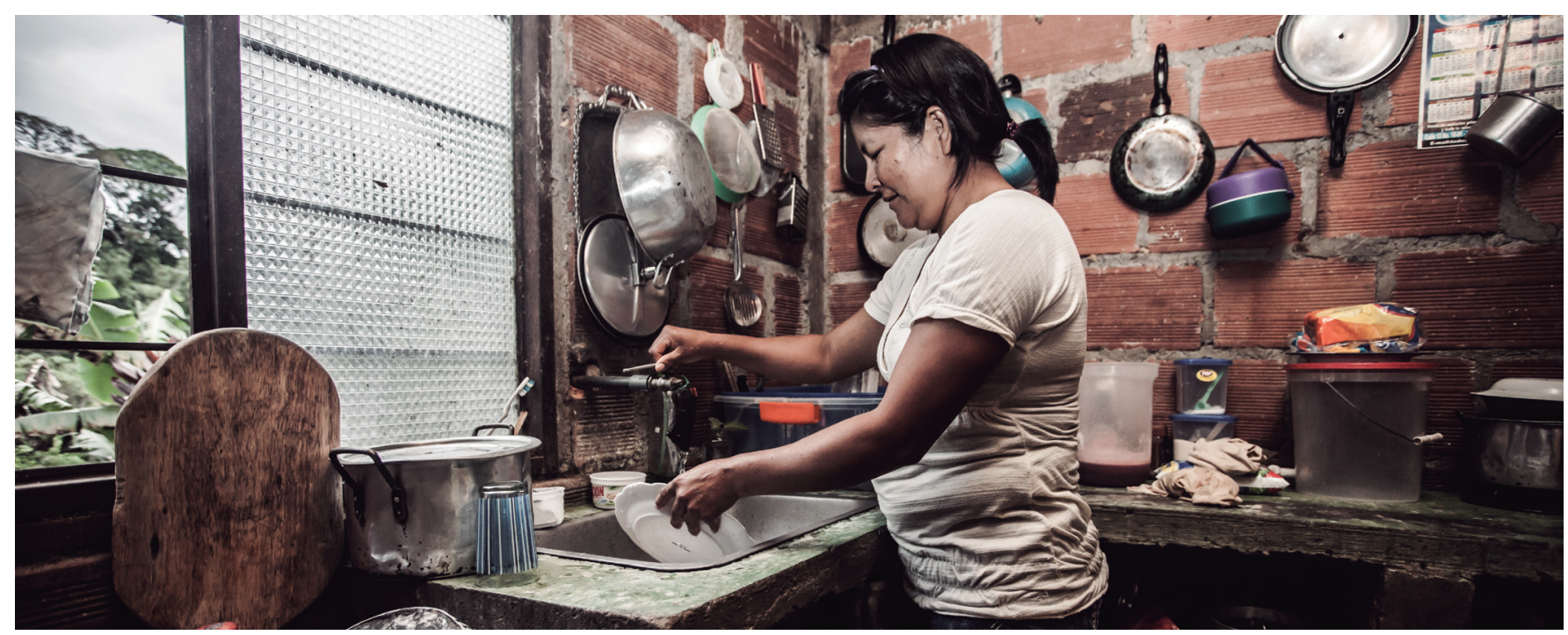

Córdoba, Quindío. Fotografía: Andrés Felipe Valenzuela. Comunicaciones y Marca-ELCA, Uniandes.

la operación y se encargaba de reparaciones mayores que ejecutaba directamente). Hasta 1987, la División de Saneamiento Básico Rural facilitó la construcción o ampliación de 2.500 acueductos, proporcionando o mejorando la calidad del servicio de agua potable a un total aproximado de dos millones de personas.

Entre 1987 y 1990, con la implementación de la descentralización en el sector de agua potable (Decreto No 077/1987) se eliminó el PASBR y se trasladó su ejecución a las dependencias departamentales, donde se reubicaron los funcionarios que hasta esa fecha manejaban el programa. Tales dependencias, sin respaldo presupuestal definido, fueron desapareciendo hasta ser eliminadas en su totalidad, perdiéndose la experiencia y conocimiento que se tenía en el manejo y promoción a nivel de la comunidad y generando un estancamiento en la capacidad del orden nacional para apoyar el sector rural tanto en sus necesidades de asistencia técnica como financiera (OPS, 1997).

Posteriormente, entre 1990 y 1998, entidades como el Fondo de Desarrollo Rural Integrado (DRI), la Caja Agraria (a través del Programa Vivir Mejor) y el Programa Nacional de Rehabilitación (PNR) desarrollaron obras de agua potable y saneamiento básico en la zona rural, cofinanciadas con los recursos de las transferencias realizadas a los municipios, llegando a ejecutar como máximo un 10\% de la inversión en el sector, es decir que el $90 \%$ restante se orientaba al sector urbano. Estas entidades y sus programas de apoyo financiero desaparecieron al finalizar la década de los noventa, de tal manera que la financiación del sector en la zona rural quedó bajo la responsabilidad casi exclusiva de los municipios.

Los municipios por su parte, habían desarrollado hasta ese momento su política de financiamiento sectorial para la zona rural, con base en las leyes que reglamentan el uso de los recursos que les transfiere la Nación: la Ley No 60 de 1993 obligaba que las inversiones en el sector de agua potable y saneamiento básico se hicieran de manera proporcional a su porcentaje de población urbana y rural; posteriormente las Leyes 715 de 2001 y 1176 de 2007 eliminaron dicha restricción, quedando a discreción de la administración municipal la asignación de recursos entre áreas urbanas y rurales.

En el periodo 2006 al 2014, el Gobierno Nacional adoptó como política pública sectorial la implementación de los Planes departamentales para el manejo empresarial de los servicios de agua y saneamiento (PDA), como un instrumento para regionalizar y fortalecer el manejo empresarial de los servicios públicos y concentrar en el nivel departamental los recursos nacionales y departamentales, al igual que los provenientes de las Corporaciones Autónomas Regionales (CAR) y en especial las transferencias a los municipios.

Estas medidas han afectado aún más la dinámica de las inversiones en la zona rural, toda vez que los planes de inversión de los PDA se enfocaron en las áreas urbanas 
y orientaron a los municipios a comprometer una parte de sus transferencias futuras para cubrir sus aportes de subsidios e inversiones principalmente en la zona urbana[1].

En julio de 2014 se expide el documento CONPES 3810 que establece la política para el suministro de agua potable y saneamiento básico en el ámbito rural, en cumplimiento a lo dispuesto por el Plan Nacional de Desarrollo (PND) 2010 -2014 Prosperidad para Todos.

Posteriormente, en el artículo 18 de la Ley 1753 de 2015[2] se ordena al Gobierno nacional definir esquemas diferenciales para la prestación de los servicios de acueducto, alcantarillado y aseo en zonas rurales, zonas de difícil acceso, áreas de difícil gestión y áreas de prestación, en las cuales por condiciones particulares no puedan alcanzarse los estándares de eficiencia, cobertura y calidad establecidos en la ley.

Así mismo se establece que la Comisión de Regulación de Agua y Saneamiento Básico (CRA) deberá desarrollar la regulación necesaria para esquemas diferenciales de prestación de los servicios de acueducto, alcantarillado y aseo. Y en el artículo 87 de esta misma ley determina que la Superintendencia de Servicios Públicos podrá definir criterios diferenciales para adelantar el control, inspección y vigilancia a los prestadores de acueducto, alcantarillado y aseo en áreas rurales.

Para dar cumplimiento a lo establecido en la Ley 1753, el Gobierno Nacional elaboró un proyecto de Decreto que busca definir esquemas diferenciales para los servicios de acueducto, alcantarillado y aseo en zonas rurales para todo el territorio nacional, el cual se encuentra en el proceso final de revisión y discusión para su expedición.

\section{Estado del arte}

De acuerdo con las cifras oficiales del DANE, la población del país en el año 2013 alcanzó los 47,1 millones de habitantes, de los cuales 11,2 millones $(23,8 \%)$ se ubican en la zona rural de las regiones Andina (46\%),

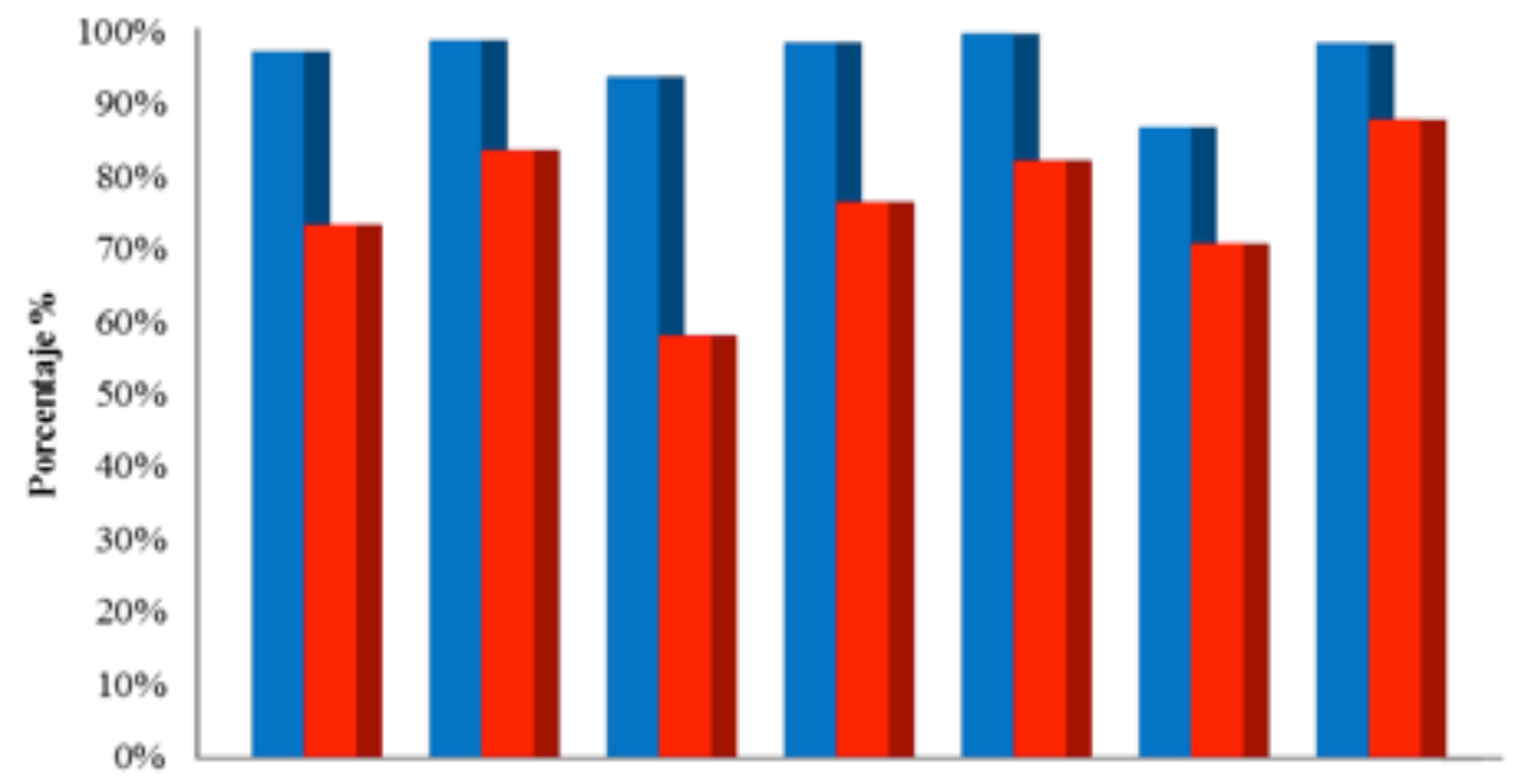

\begin{tabular}{|l|c|c|c|c|c|c|c|}
\cline { 2 - 8 } \multicolumn{1}{c|}{} & TOTAL & Antioquia & Caribe & Oriental & Central & Pacifica & $\begin{array}{c}\text { Valle del } \\
\text { Cauca }\end{array}$ \\
\hline - Acueducto urbano & $97 \%$ & $99 \%$ & $94 \%$ & $98 \%$ & $99 \%$ & $87 \%$ & $98 \%$ \\
\hline - Acueducto nural & $73 \%$ & $83 \%$ & $58 \%$ & $76 \%$ & $82 \%$ & $71 \%$ & $88 \%$ \\
\hline
\end{tabular}

Grafico 1. Fuente: Elaboración propia. 


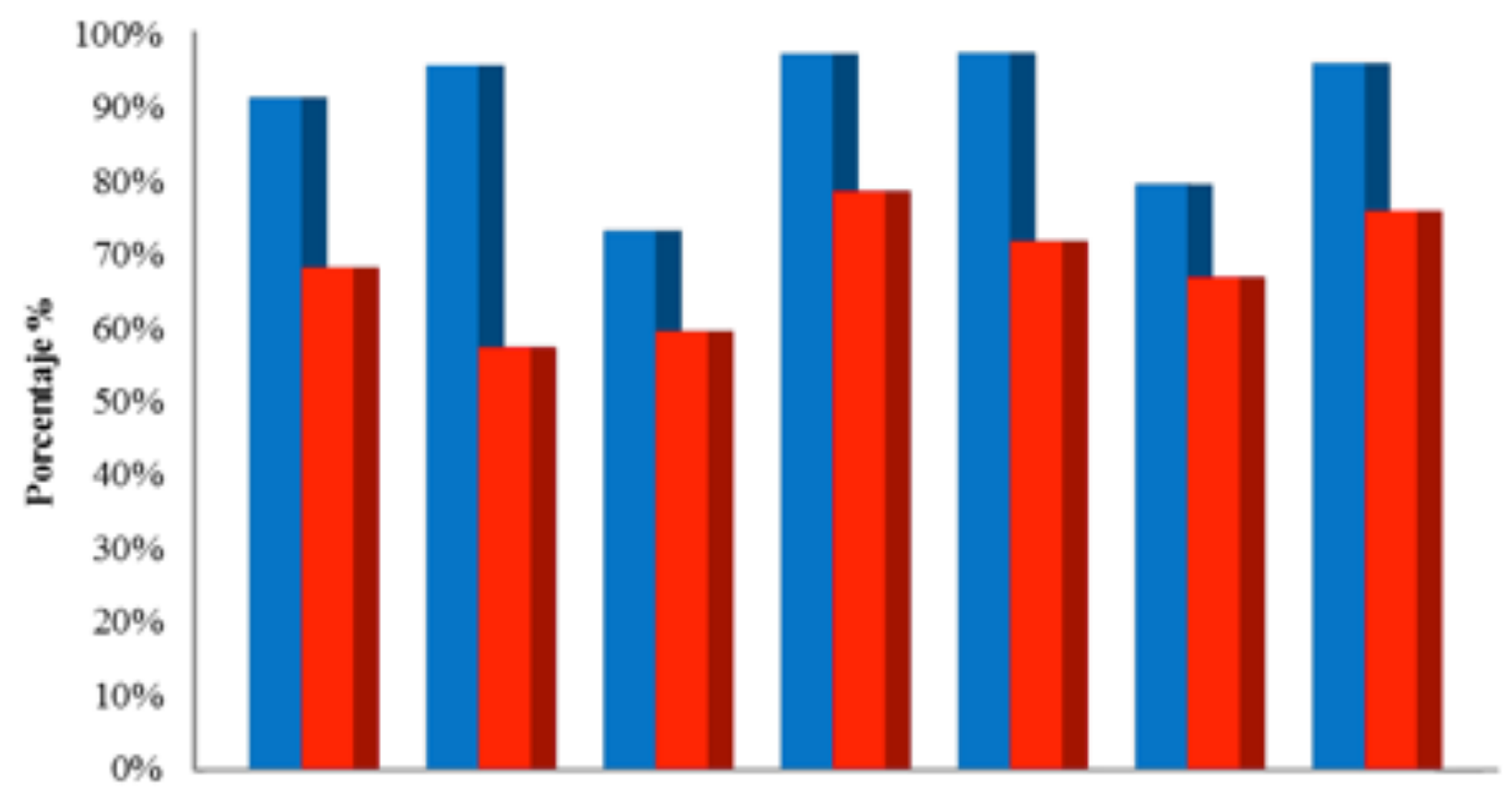

\begin{tabular}{|l|c|c|c|c|c|c|c|}
\cline { 2 - 8 } \multicolumn{1}{c|}{} & TOTAL & Antioquia & Caribe & Oriental & Central & Pacifica & $\begin{array}{c}\text { Valle del } \\
\text { Cauca }\end{array}$ \\
\hline - Alcantarillado urbano & $91 \%$ & $96 \%$ & $73 \%$ & $97 \%$ & $97 \%$ & $79 \%$ & $96 \%$ \\
\hline - Alcantarillado rural & $68 \%$ & $57 \%$ & $60 \%$ & $79 \%$ & $72 \%$ & $67 \%$ & $76 \%$ \\
\hline
\end{tabular}

Grafico 2. Fuente: Elaboración propia.

Caribe $(23 \%)$ y Pacífica $(22 \%)$. De estos, un $23 \%$ de la población se concentra en áreas nucleadas y el 77\% en áreas dispersas. De acuerdo con los resultados de la Gran Encuesta Integrada de Hogares (GEIH) de 2012, las diferencias entre las coberturas de acueducto en áreas urbanas y rurales llegan a los 24 puntos porcentuales, mientras que en alcantarillado la diferencia es de 23 puntos. (Ver gráficos 1 y 2), con una mayor diferencia en la región Caribe.

De igual manera existe un desequilibrio marcado en cuanto a la calidad del agua que se suministra a la población en el área rural, mientras que en área urbana el Índice de Riesgo de Calidad del Agua (IRCA) promedio en 2012 fue del $13,2 \%$, correspondiente a nivel de riesgo bajo, en el área rural este valor alcanzó el 49.8\%, es decir en nivel de riesgo alto .

De acuerdo con el CONPES 3810, las regiones en donde se hace un mayor esfuerzo de inversión y acciones para mejorar el acceso a agua potable y saneamiento básico, son las que presentan unas menores tasas de mortalidad y morbilidad por EDA en menores de 5 años.

Por otra parte, la evaluación de los Objetivos de Desarrollo del Milenio (ODM) en Colombia arroja un incumplimiento en la meta de reducción a la mitad del déficit existente a 1990 en las coberturas de agua potable y saneamiento en el área rural: en 2015 la cobertura de agua potable debía llegar al 84,4\% y en saneamiento al $70,4 \%$, en tanto que los resultados alcanzados fueron del $73,8 \%$ y $67,9 \%$ respectivamente (Ver gráficas 3 y 4 ).

Con la aprobación de los Objetivos de Desarrollo Sostenible (ODS) por parte de la Asamblea de las Naciones Unidas en septiembre de 2015, las nuevas metas al año 2030 se han hecho mucho más exigentes.

Por ejemplo, El ODS No 6 se propone "Garantizar la disponibilidad de agua y su gestión sostenible y el saneamiento para todos", estableciendo 6 metas que incluyen la universalidad de estos servicios en el año 2030. 


\section{Cobertura - Acceso a fuentes de agua mejoradas}

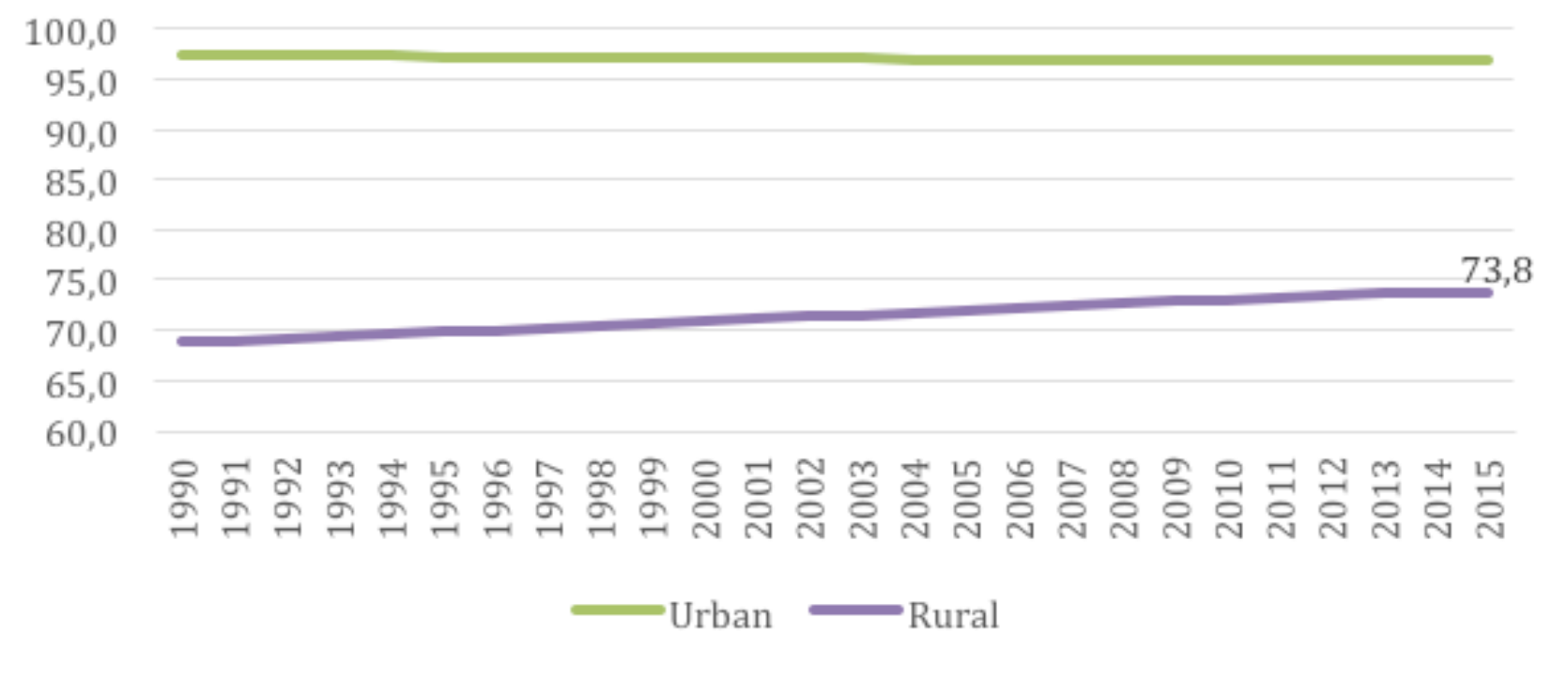

Grafica 3. Evolución de las coberturas en agua potable (1990-2015). Elaboración propia.

\section{Cobertura - Acceso a Saneamiento Mejorado}

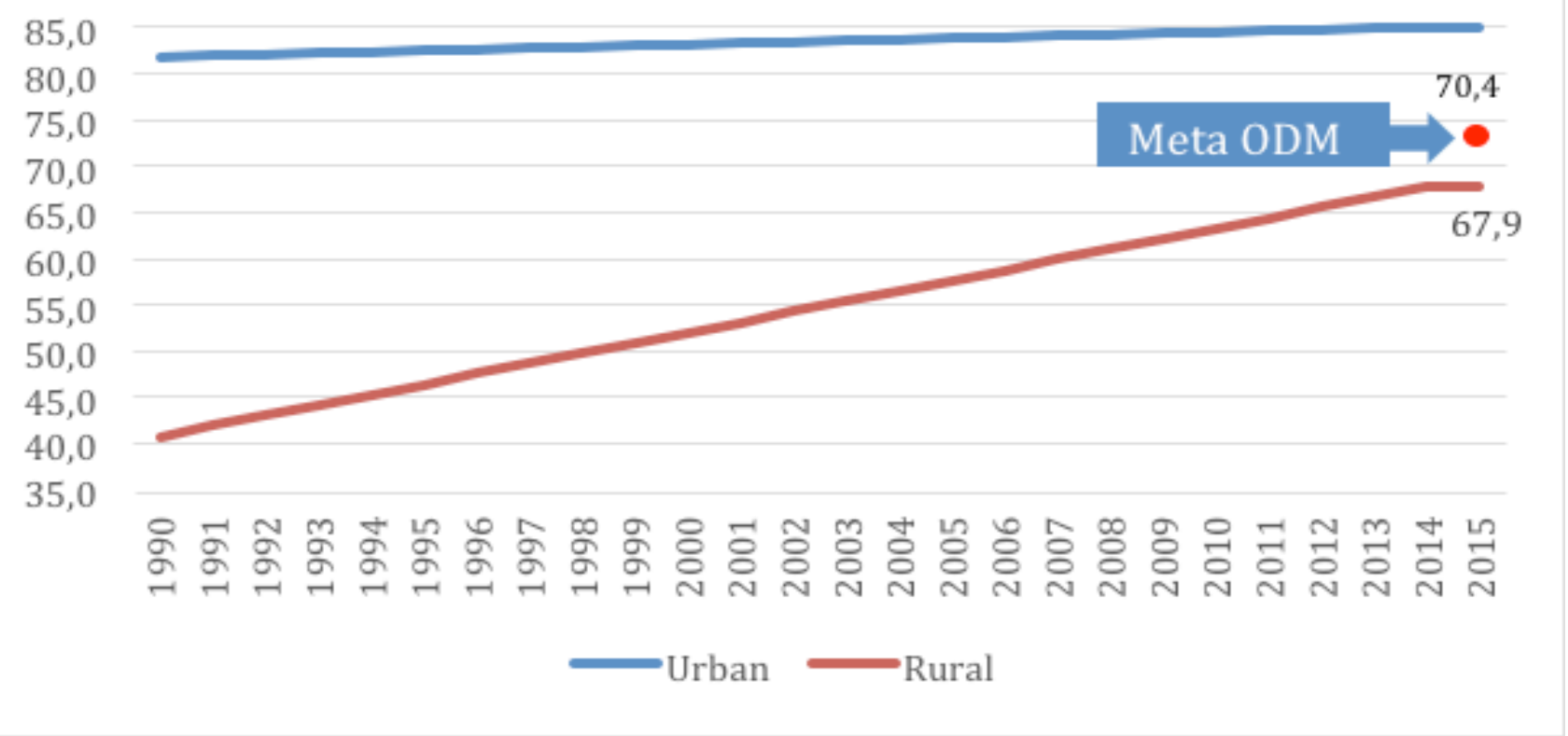


Si se mantuvieran los niveles de inversión y los esquemas de intervención de los últimos 25 años en el país, los resultados en el año 2030, frente a la meta de cobertura universal del servicio, serían los que se muestran en las siguientes gráficas:

\section{Proyección - Acceso a fuentes de agua mejorada}

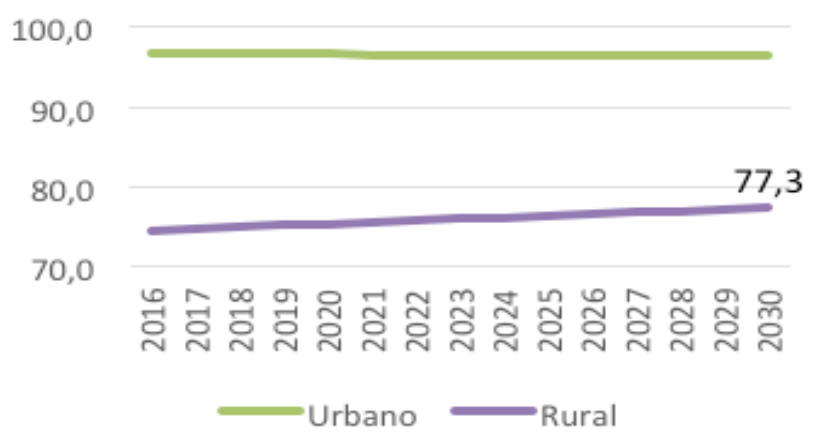

\section{Proyección - Acceso a saneamiento mejorado}
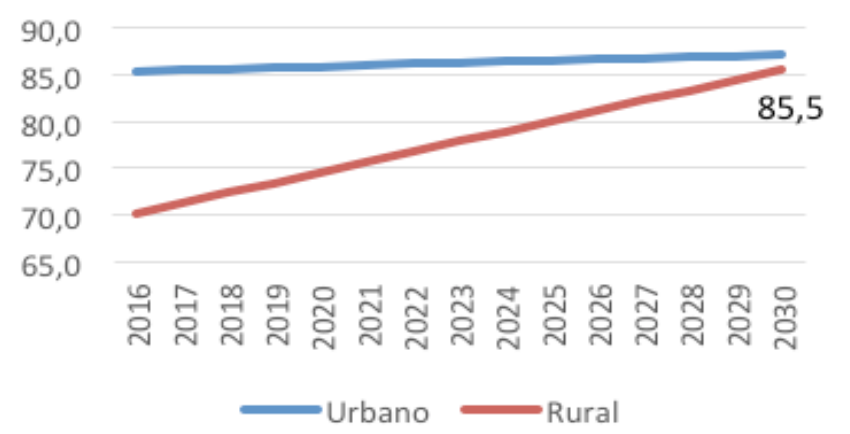

Proyecciones con tendencia lineal, aplicando el método de los mínimos cuadrados. Elaboración propia.

\section{Conclusiones}

Más allá de la inequidad que muestran los indicadores de acceso, frente a las áreas urbanas, el sector de agua y saneamiento en el ámbito rural presenta grandes retos y desafíos que demandan un esfuerzo y un trabajo continuo de todas las instituciones. A continuación se relacionan los principales problemas y desafíos identificados:

\section{Inadecuado marco normativo:}

Cerca de 20 mil organizaciones comunitarias y sin ánimo de lucro que prestan servicios de agua y saneamiento en el área rural del país, carecen de autorización legal para suministrar estos servicios. La Ley 142 de 1994 autoriza la prestación de estos servicios por parte de "Organizaciones Autorizadas" sin precisar que las comunidades organizadas puedan hacer parte de esta categoría.

Por otra parte, según la Superintendencia de Servicios Públicos, las comunidades organizadas deben ser consideradas Empresas de Servicios Públicos en los términos de la Ley 142 de 1994 y en consecuencia cuando un municipio quiera entregar la prestación de los servicios de agua y saneamiento en el área rural, debe hacerlo por medio de una licitación pública (Parágrafo del Art. 31 de la Ley 142 de 1994). Aun cuando la Corte Constitucional no comparte esta opinión, es evidente que existe un vacío legal que el Gobierno Nacional debe gestionar y atender de manera prioritaria.

La regulación, el control y la vigilancia de los servicios en el área rural requieren una reglamentación especial en aspectos como: i) cobro de derechos de conexión; ii) indicadores de gestión y resultados; iii) sistemas alternos de medición de los consumos, entre otros.

\section{Información deficiente}

El País no dispone de un sistema de información que permita establecer con algún grado de precisión el monto de los recursos financieros invertidos en el incremento de coberturas o el mejoramiento de la calidad de los servicios de agua potable y saneamiento en el sector rural, ya que los datos existentes mezclan la inversión en infraestructura que se realiza en las áreas urbanas y rurales. Tampoco existe información actualizada de los prestadores de agua y saneamiento, ni de la calidad de los servicios con que cuenta la población rural en el País. Menos del 2\% de las muestras que toman las autoridades sanitarias para vigilar la calidad del agua en el País, son tomadas en áreas rurales. Es muy escaso el registro de prestadores rurales en el SUI de la Superintendencia de Servicios Públicos y sus acciones de control desestimulan la formalización de los pocos prestadores registrados. 


\section{Débil capacidad institucional de los entes territoriales}

Pocos municipios, distritos y departamentos cuentan con estructuras institucionales o programas específicos para llevar a cabo las labores que les corresponden de planificación, asistencia técnica y ejecución de proyectos de infraestructura de agua potable y saneamiento básico para el área rural.

Desarticulación de acciones en el nivel nacional

No existe una instancia en el nivel nacional que articule las acciones que se ejecutan en el área rural por parte de los distintos Ministerios, entidades de Gobierno y organismos de cooperación internacional.

\section{Participación de la comunidad en los proyectos}

La metodología empleada en el diseño y construcción de los proyectos de agua potable y saneamiento básico para el sector rural se concentra en la elaboración de los estudios de ingeniería y la construcción de las obras, sin tener en cuenta los conocimientos, necesidades y preferencias de la comunidad.

Atomización de prestadores

Según las cifras del Inventario Sanitario Rural, hacia el año 2000 en el País existían 11.552 entidades prestadoras del servicio en el área rural. Esta fuerte atomización de prestadores en la zona rural limita la capacidad de cubrir los costos que genera su operación individual y complica las funciones de asistencia técnica, capacitación, regulación, vigilancia y control.

Algunas particularidades que explican este mercado atomizado son:

i) las características geográficas de las áreas rurales

ii) las grandes distancias que con frecuencia separan las veredas y pequeños centros poblados.

iii) las dificultades técnicas y económicas para la construcción de sistemas regionales de abastecimiento de agua y saneamiento básico

iv) la baja valoración del servicio y reducida capacidad de pago de la población rural.

v) la importancia que dan los usuarios a las ventajas de la cercanía a su prestador para la solución pronta de sus peticiones, quejas y recursos, la atención personalizada, la facturación, el cobro local, la oportunidad de la información y la pronta reparación de fallas y daños en el servicio, entre otros aspectos.

En este sentido, existen múltiples evidencias de sistemas administrados por comunidades organizadas que operan con criterios de autosostenibilidad y eficiencia. Sin embargo, las cifras del ISR indican que solo un $23 \%$ de estos prestadores cuentan con personería jurídica;

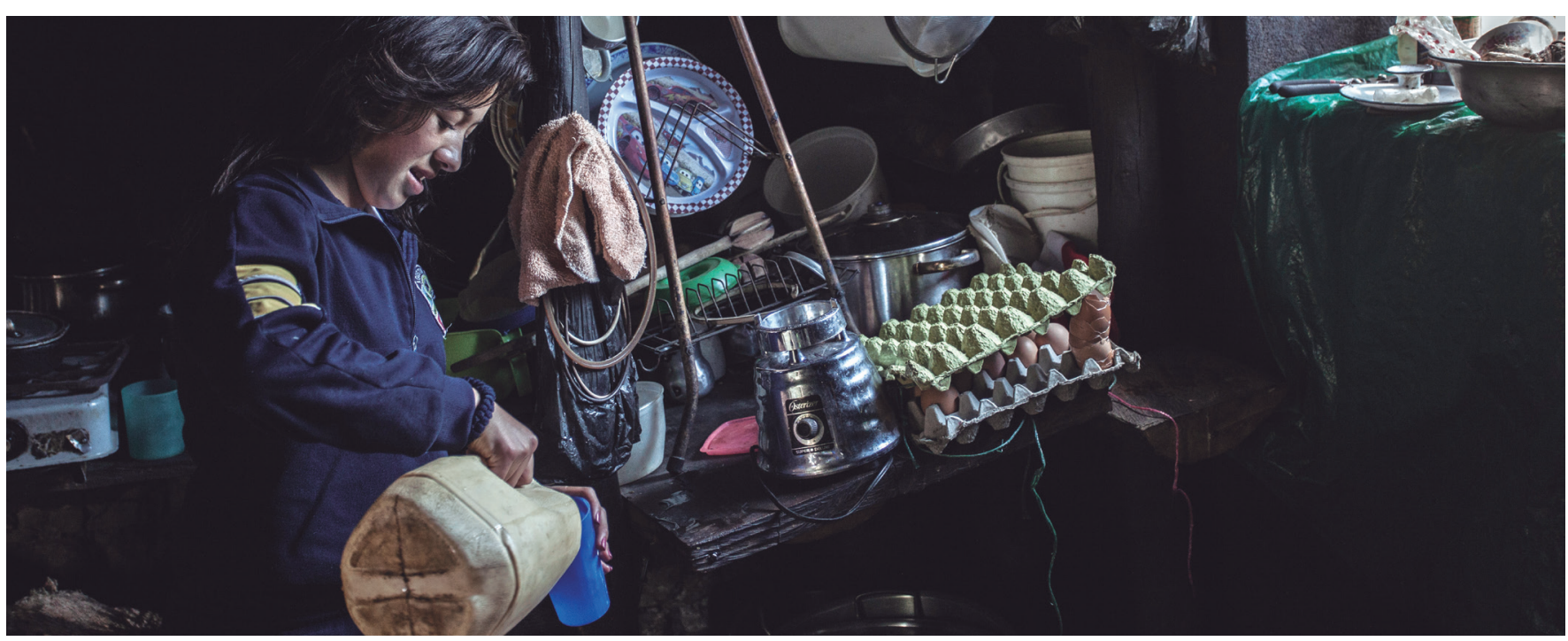

Simijaca, Boyacá. Fotografía: Alejandro Gómez. Comunicaciones y Marca-ELCA, Uniandes. 
31,8\% emiten algún tipo de factura; $7.7 \%$ cuentas con estudios de costos y tarifas; solo 6\% llevan contabilidad y apenas un 10,5\% cuentan con micromedición.

\section{Infraestructura inadecuada para el ámbito rural}

Son frecuentes los conflictos por el uso del agua en el área rural: el consumo humano y las actividades productivas compiten por la calidad del agua requerida en cada caso y por el volumen disponible para su uso, especialmente en sistemas de uso múltiple.

Adicionalmente, los estudios y diseños se orientan a la utilización de tecnologías convencionales de difícil operación y elevados costos que afectan su sostenibilidad en el largo plazo. Se incentiva el uso de soluciones colectivas de saneamiento, con énfasis en alcantarillados convencionales y plantas complejas para el tratamiento de aguas residuales y se desestimula el uso de soluciones individuales de más bajo costo y más adecuadas para el ámbito rural.

Este panorama precisa medidas de fondo que den un nuevo impulso al sector de agua y saneamiento rural en el País y se constituyen en un verdadero desafío para el Estado como un todo frente a los Acuerdos pactados en la Habana y las nuevas funciones de la Agencia de Desarrollo Rural.

Una nueva institucionalidad con recursos frescos es requerida de manera urgente, si se pretende como objetivo cerrar la brecha existente entre el área urbana y rural y saldar -en parte- la deuda histórica que tiene el País con el campo.

\section{Referencias}

[1] Los PDA que han viabilizado proyectos para la zona rural son: Atlántico, Caldas, Cauca, Córdoba, Cundinamarca, Guainía, Guajira, Huila, Magdalena, Meta, Nariño, Risaralda y Vaupez, con un monto total de recursos que ascienden a 99 mil millones.

[2] Plan Nacional de Desarrollo 2014-2018 "Todos por un nuevo país"

[3] De acuerdo con el DANE, en la zona rural se distinguen dos clases de asentamientos: los Centros poblados o población nucleada, concentrada en caseríos o conjuntos de por lo menos 20 viviendas separadas por paredes, muros, cercas o huertas; y el segundo se denomina fincas y viviendas dispersas (población dispersa) separadas, entre otros, por áreas cultivadas, prados, bosques, potreros, carreteras o caminos.

[4]Tomado del Informe" Estado de la Vigilancia de la Calidad del Agua para Consumo Humano en Colombia" Instituto Nacional de Salud, 2012. Pág 7.

[5] Fuente: Programa Conjunto de Monitoreo (JMP) de OMS y UNICEF, http://www.wSsinfo.org/data-estimates/tables/. Mayo 23 de 2016

[6] Según las cifras del Inventario Sanitario Rural, hacia el año 2000 en el País existían 11.552 entidades prestadoras del servicio en el área rural. A partir de esta cifra se estima que a 2016 pueden existir cerca de 20.000 prestadores.

[7] Circular SSPD 034 de 2010, numeral 6.

[8] Sentencia C-741/03 del 28 de agosto de 2003 